\title{
CEYLONS WILDE UND ZAHME ELEFANTEN
}

\author{
VON PALL WIRZ
}

7 Abbildungen

Ceylon und die Elefanten! - Irgendwie gehörten sie schon immer zusammen, und wenn man von Ceylon spricht, so denkt man unwillkürlich auch an seine Elefanten, die den undurchdringbaren Dschungel, der noch immer viele Quadratkilometer der Insel bedeckt, als ihren ureigenen und alleinigen Tummelplatz haben, wo sie außer dem Menschen, der sich hin und wieder in ihr Bereich wagt, nichts zu fürchten brauchen.

Und weiterhin denkt man auch an die vielen Hunderte von Elefanten, die vor gut zweieinhalb Jahrtausenden schon gezähmt und dazu verwendet wurden, dem Menschen dienlich zu sein, allerlei nützliche Arbeit zu verrichten, aber auch um an Kriegszügen, prunkvollen Festlichkeiten der Könige und an religiösen Umzügen teilzunehmen. Alles das gibt es auch heute noch, sowohl wilde wie auch gezähmte Elefanten, wenn auch ihre Zahl langsam, aber unaufhaltsam zurückgeht und der Elefantenfang ein seltenes Schauspiel geworden ist.

\section{Terminologie und Klassifikation}

Elephas indicus ist der zoologische Name dieses Dickhäuters. Aliya nennt der Singhalese den Elefanten; daneben kennt man aber weiterhin die Bezeichnung eta für das männliche und etini für das weibliche Tier. Demgegenüber besitzen die Tamilen bloß die eine Bezeichnung yanne, die sowohl für das männliche und auch das weibliche Tier gilt. Im Sanskrit, Pali und Hindustani heißt der Elefant gaja. Komban ist weiterhin bei den Singhalesen die Bezeichnung für große Elefanten mit gut entwickelten Stoßzähnen, für die besondere Bezeichnungen wohl nötig erschienen.

Von dem auf dem indischen Festland vorkommenden Elefanten unterscheidet sich der ceylonesische vor allem dadurch, daß jener im allgemeinen große Stoßzähne aufweist, während auf Ceylon höchstens fünf Prozent aller männlichen Tiere solche besitzen. Bei allen andern bleiben die Stoßzähne klein und unscheinbar. Die längsten Stoßzähne eines ceylonesischen Elefanten befinden sich im Tempel von Lanka Tilaka, zwölf Meilen südlich von Kandy. Sie sind, der Krümmung nach gemessen, 1,90 m lang.

$\mathrm{Daß}$ die indischen Elefanten größere und stärker entwickelte Stoßzähne haben, wird von seiten der Zoologen dem Umstand zugeschricben, daß der Boden Ceylons kalkarm, derjenige Indiens, so wenigstens in manchen Gegenden, reich an Kalk ist. Dancben gibt es zwischen den hier und dort vorkommenden Elefanten noch eine Reihe weiterer Unterschiede, die aber weniger in die Augen springen.

Die Eingeborenen Ceylons pflegen die in Südasien vorkommenden Elefanten nach bestimmten Gesichtspunkten in eine Anzahl von Klassen oder Gruppen einzuteilen, die sich nicht mit denjenigen der Zoologen decken.

Von manchen europäischen Forschern werden die verschicdenen Klassen oder Rassen, in welche die Eingeborenen Ceylons und auch diejenigen Indiens die Elefanten einteilen und für welche die Singhalesen ganz allgemein die Bezeichnung djati gebrauchen, vielfach mit den Kasten der Menschen verglichen, was aber insofern wenig zutreffend sein dürfte, als die Klassierung der Eingeborenen auf rein äußcrlichen Merkmalen beruht. So äußert sich zum Beispiel Haughton hinsichtlich der Einteilung der Ceylon-Elefanten folgenderweise: "The people of Ccylon, universally recognize 'caste' in clephants; projecting their own ingrained ideas into other animals, especially the lordly elephant. The highest caste elephants are what are called 'white' elcphants; they are covered with large irregular pinkish-white blotches on the head, ears, and trunk, and are greatly prized by the pcople. A good caste clephant is of well-shaped head, strong compact body, with stout legs and feet; latter of good diameter. The circular fore-feet should contain five good-sized handsome nails. His cyes should be the colour of honey. Low-caste clephants are lanky, long-legged animals, with small fect and black skin."

Demnach soll das Hauptgewicht bei der Bewertung eines Elefanten vor allem auf die Färbung der Haut und der Augen gelegt werden, was in gewissen Fällen, und von rein ästhetischem Gesichtspunkt aus betrachtet, zutreffen mag. Doch spiclen gerade diese Merkmale bei der Einteilung der Elefanten in die verschiedenen djati gar keine Rolle. Sie erfolgt vielmehr nach ganz anderen Gesichtspunkten, wie der allgemeinen Körpergröße, der Form des Kopfes, der Länge der Beine, des Rüssels und des Schwanzes, der Zahl der Zehen, der Körperbehaarung und andern Merkmalen. 
Die Eingeborenen Ceylons unterscheiden zehn djati von Elefanten: Tamba, Gangeya, Kantje, Kalava, Pingala, Gandamana, Uposata, Pandara, Sadhanta (oder Tjadhanta) und Hema. Von diesen kommen jedoch bloß die vier erstgenannten auf Ceylon vor.

Die vier ceylonesischen Elefantenrassen unterscheiden sich ausschließlich durch Größe, Fuß-, Ohrund Kopfform, Größe von Rüssel und Schwanz und Behaarung, wobei natürlich bloß die vollkommen ausgewachsenen Tiere in Betracht zu ziehen sind. Am größten sind die Tamba; auf sie folgen die Gangeya, die Kantje und die Kalava.

\section{Zahl, wilde und gezähmte Elefanten}

Über die Zahl der Elefanten liegen bloß hinsichtlich der gezähmten Tiere einigermaßen zuverlässige Angaben vor.

Nach Aussage der Eingeborenen soll sie ungefähr 1500 betragen, was aber zu hoch gegriffen ist. Der dritte Teil dürfte der Wirklichkeit entsprechen. Die gezähmten Elefanten leben vor allem in den Plantagengebieten, also in den zentralen Provinzen der Insel, wo sie beim Reinigen des Dschungels und beim Abschleppen der Baumstämme wertvolle Dienste leisten.

Über die Zahl der wilden Elefanten gehen die Mutmaßungen weit auseinander. Meistens hört man von 1500 bis zu 2000 Tieren sprechen, während andere diese Zahlen auf das Doppelte oder gar Dreifache veranschlagen. Sie halten sich vor allem in den gebirgigen, schwer zugänglichen südöstlichen

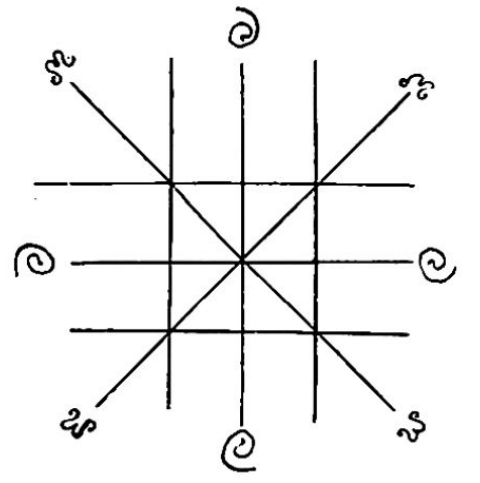

Abb. 1. yantra Gebieten der Insel auf, so vor allem in der Provinz $U$ wa und in dicht bewaldeten, nur sehr spärlich besiedelten Waldgebieten zwischen den beiden alten Hauptstädten Anuradhapura und Polonnaruwa, wo sie noch größere Herden bilden.

$\mathrm{Da} \beta$ die Zahl der Elefanten früher eine bedeutend höhere war, nun langsam, aber stetig zurückgeht, versteht sich wohl von selbst, obschon das Abschießen und Einfangen von Elefanten heute streng geregelt ist und Massenfänge, sogenannte Kraalings, nicht mehr vorkommen. In früherer Zeit muß der Reichtum an Elefanten auf dieser Insel nach Reisewerken und Beschreibungen ein ganz erheblicher gewesen sein.

Über die Gefährlichkeit der wilden Elefanten ist von jeher viel berichtet worden, doch widersprechen sich auch da die Aussagen oft sehr. Natürlich beurteilt der Europäer, der über dieses Thema spricht oder schreibt, aber in den wenigsten Fällen jemals einem wilden Elefanten begegnet ist, diese Angelegenheit anders als der im Dschungel lebende Eingeborene, der sozusagen ständig in Gefahr schwebt, von einem wilden Elefanten angenommen zu werden.

Die Gefahr, auf eine Elefantenherde oder einen Einzelgänger zu stoßen, ist jedoch gering geworden. Es wissen auch blo $\beta$ die in den in Frage kommenden Gebieten lebenden Eingeborenen anzugeben, wo eventuell eine Elefantenherde noch anzutreffen ist. Die Gefährlichkeit betrifft vor allem die berüchtigten Einzelgänger oder Rogue. Vielfach wird behauptet, daß ein solches Tier aus seiner Herde wegen Krankheit oder sonst aus einem Grunde ausgestoßen worden sei, was jedoch nicht richtig ist. Ebensowenig trifft zu, daß diese Tiere von Haus aus wild und bösartig seien. Es handelt sich jedoch immer um besonders mutige und unternehmende Tiere, die sich auch in die Nähe der Niederlassungen wagen und dadurch gefährlich werden können. Der Eingeborene, der in der Regel keine oder nur mangelhafte Schußwaffen besitzt, fürchtet diese Tiere ungemein. Einem Angriff gegenüber weiß er sich eigentlich bloß durch ein geeignetes yantra und mantra zu schützen.

Ein solches yantra (Abb. 1) wird bei einer Wanderung durch den Dschungel mit Betelkalk, den man meistens bei sich hat, eventuell auch mit Kohle auf die beiden Handflächen gezeichnet, und man spricht hierauf in die offenen Handflächen das folgende mantra, und zwar siebenmal: "Om namo! Sala Mahanura Asupala - kumarige bade upan siddahasa pirivärati sidda Maha- sohona ira-kanda, -sanda -kanda, ira-mudun velava alata matura me matreng alata matura käli-aliya matura. - ata vahanova. - Esvaha!» Frei übersetzt: «Om, Buddha die Ehre! Im Bergland von Mahanura (dem großen Land) gebar die Prinzessin Asupala 17 Yakka, welche die Gefolgschaft von Maha- sohona (dem großen Friedhofteufel) bildeten. Wenn die Sonne hinter den Bergen steht oder mittags, kurz, bei jeder Gelegenheit spreche man dieses mantra auf die beiden Handflächen zur Abwehr der Dschungelelefanten. Hierauf schließe man die Handfächen. Dies (d. h. das yantra) sei gut!" Hat man das mantra gesprochen, so legt man die Handfächen zusammen und setzt in dieser Weise seinen Weg fort, um im Moment, wo man einen wilden Elefanten erblickt, ihm die Handflächen mit dem yantra entgegenzuhalten.

Auch gegen das Zerstörtwerden der Pflanzungen durch dic Dschungelelefanten wendet man yantra an. Man befestigt diese an den Bäumen oder in die Erde gesteckten Stöcken, nachdem man sie zuvor mit einem geeigneten mantra besprochen hat. 
Es gibt Leute, die sich ausschließlich, also beruflich, mit der Anfertigung solcher yantra befassen; doch stehen sie nicht in sonderlich hohem Ansehen.

\section{Fang und Zähmung}

Seit den ältesten historisch belegten Zeiten wird der Elefantenfang auf Ceylon nach zwei verschiedenen Methoden betrieben, die schon oft beschrieben wurden. Die eine ist der Einzelfang, wobei durch wenige mutige und mit den Verhältnissen vollkommen vertraute Menschen aus einer Herde heraus ein junges Tier einzufangen versucht wird. Die andere Methode, das Kraaling, ist ein sorgfältig vorbereiteter Massenfang, der in früheren Zeiten alle paar Jahre einmal durch den König, späterhin durch die Regierung, veranstaltet wurde. $\mathrm{Daß}$ bei ihm alle möglichen Tiere, junge und alte, Weibchen und Männchen, unterschiedslos in den Kral getrieben und dann einzeln eingefangen wurden, wobei dann aber regelmäßig ein mehr oder weniger großer Prozentsatz der Tiere sich gegenseitig zugrunde richtete oder wegen seines Benehmens totgeschossen werden mußte und daß ein weiterer großer Prozentsatz der eingefangenen Tiere sich späterhin zur Zähmung und Abrichtung vollkommen untauglich erwies, sei hier nur nebenbei er-

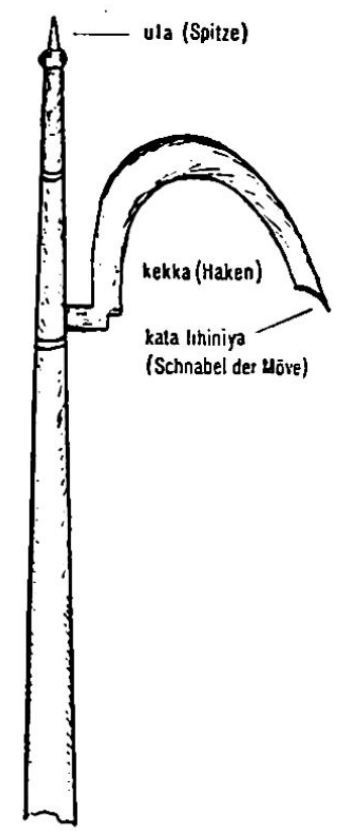
wähnt. So wird ein solches Kraaling heute nur noch sehr selten durchgeführt. Für den Einzelfang ist für die Dauer von drei Monaten und den Fang eines einzigen Tieres eine Lizenz von 1000 Rupien zu zahlen, was ebenfalls dazu beiträgt, daß nur sehr wenige sich darum bewerben.

Meistens übernimmt der Mann, der einen Elefanten gefangen hat, auch dessen Zähmung. Nach einigen Tagen oder wenigen Wochen hat er sich in der Regel so an seinen Pfleger gewöhnt, daß er alles Genießbare entgegennimmt und sich ohne weiteres betasten läßt. Natürlich bleibt er zunächst noch gefesselt; aber recht bald ist er so weit, $\mathrm{da} ß$ der Mann den Versuch unternehmen kann, von einem Gestell oder Baum herab auf seinen Rücken zu gelangen. So wird er so rasch wie möglich daran gewöhnt, daß jemand auf seinem Rücken sitzt. Erst wenn er auch dies geschehen läßt, beginnt man seine Fesseln zu lösen, und dann wird er, meist in Begleitung eines oder zweier anderer zahmer Elefanten, nach dem Dorf gebracht.

Bei der Zähmung der Elefanten spielt als einziges Gerät oder Instrument der Elefantenstachel (singhalesisch hendua) eine Rolle, der auch späterhin in der Hand des mahut von Bedeutung ist. Es ist dies ein uraltes Gerät, das zweifellos indischen Ursprungs ist. Der Elefantengott Ganesha (oder Ganapati, wie er auf Ceylon meistens genannt wird) trägt es als Attribut und Waffe in einer seiner zahlreichen Hände, und so wird auch diese Gottheit stets mit einem solchen Elefantenstachel in einer der beiden Linken dargestellt (Abb. 2).

Man unterscheidet an diesem Gerät die eigentliche Spitze (ula) und den Haken (kekka), dessen Ende häufig sichelförmig gestaltet ist und mit dem Schnabel eincr Möve (kata lihiniya) verglichen und auch so benannt wird. Im übrigen kann jedoch der hendua sehr verschieden gestaltet, d. h. der Haken groß oder klein, der Schaft lang oder kurz sein. Oftmals besteht das Ganze aus zwei oder drei zusammenschraubbaren Teilen.

Es sind gewisse Berufsleute (Schmiede), die sich auf die Herstellung des hendua verstehen. Nicht jeder gewöhnliche Schmied versteht sich darauf. Denn die Herstellung dieses Gerätes erfolgt nach ganz bestimmten Vorschriften, die nur Berufsleuten bekannt sind und von ihnen geheimgehalten werden. Überdies müssen bei der Herstellung eines hendua gewisse Vorsichtsmaßregeln beachtet, mantra gesprochen und der Gottheit $G$ anesha ein kleines Opfer dargebracht werden. Erst nachdem dies geschehen, schreitet man zur Herstellung des Gerätes, womit jedoch nur an einem zum voraus zu bestimmenden günstigen Tage begonnen werden kann.

Das in Frage kommende Material ist natürlich Eisen; doch muß für die äußerste Spitze ein Stück Meteoreisen (henakambi) genommen werden, wie es in früherer Zeit auch bei der Anfertigung von Lanzenspitzen, Messer- und Schwertklingen und auch der Schreibstifte, mit denen man die Schriftzeichen in die Palmblattstreifen einritzte, geschah. Der aus gewöhnlichem Eisen, ohne Zutun von Meteoreisen, hergestellte hendua wärc untauglich oder doch recht minderwertig. 


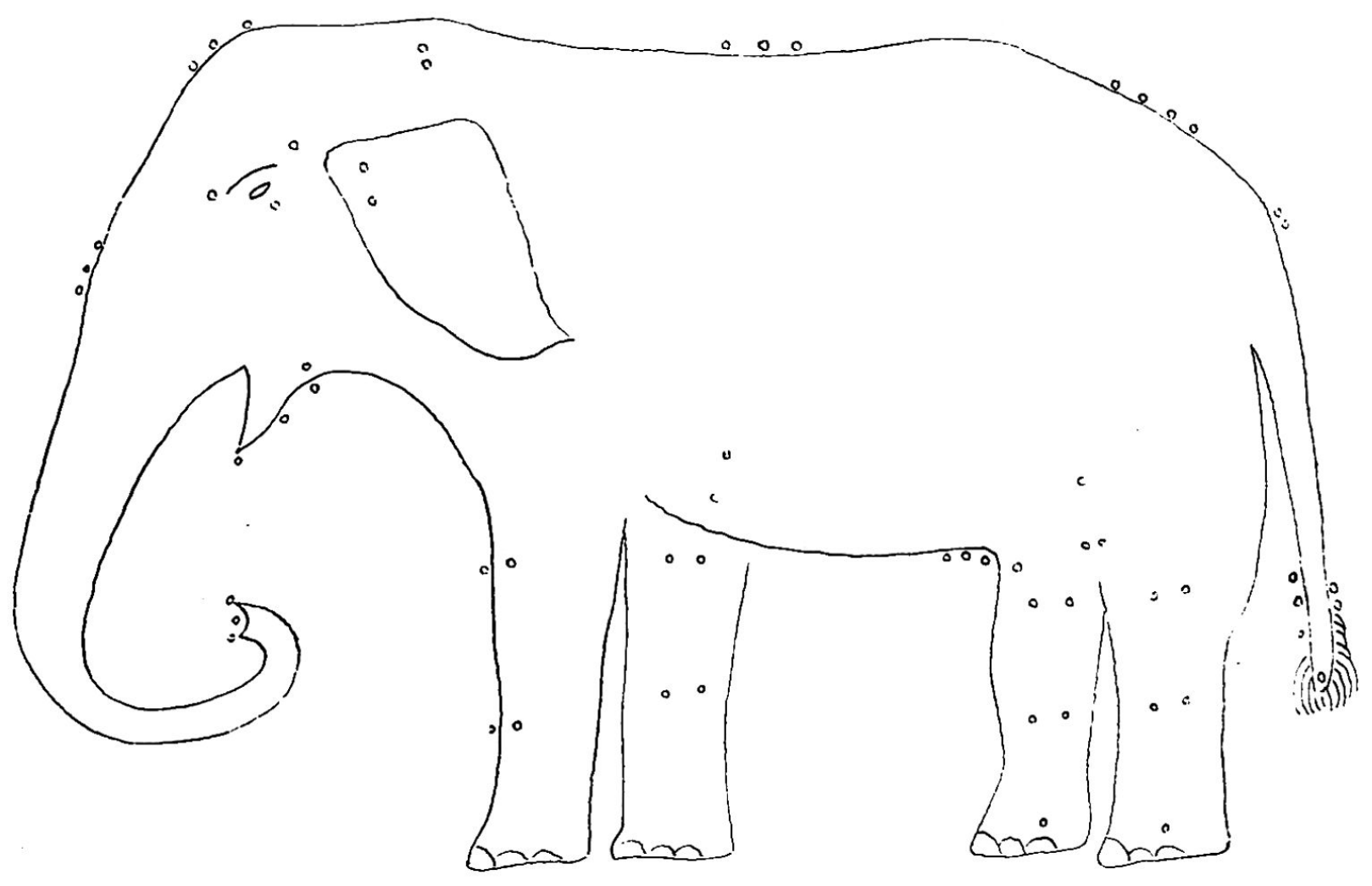

Abb. 3. Die nila (die empfindlichen Stellen) des Elefanten

Ist das Gerät soweit fertig, so müssen die Spitze und der Haken unter Hersagen einer gewissen mantra in eine "Medizin" getaucht werden, damit der hendua späterhin auch seinen Zweck zu erfüllen vermag. Eine solche "Medizin» besteht aus den folgenden Bestandteilen: bulat pilala, Loranthus (ein Parasit, der auf der Betelpfefferpflanze gedeiht); murunga pilala, ein Parasit von Moringa pterygosperma; kiral pita, Galle des Lappenkiebitzes (Lobivancllus indicus); katusa kara valaya, Kamm der Kammeidechse (Gonocephalus); divi tel oder koti tel, Fett des Leoparden oder des Tigers. Sie werden zerkleinert und in einem Topf mit etwas Wasser gekocht. Hierauf wird der Haken mehrmals in den heißen Brei getaucht, wobei das folgende mantra gesprochen wird: "Om srin hin lan hara- hara srigah-srigah srivah; esvaha.»

Der hendua dient, wie gesagt, sowohl zum Stechen, also Reizen des Dickhäuters, wie auch zum Führen oder Ziehen, indem man den Haken an irgendeiner Stelle des Körpers ansetzt. Dabei weiß der mahut, der von klein auf mit den Elefanten umzugehen gelernt hat -- es sind dies Leutc ciner besondern, nicht besonders hochstehenden Kaste --, ganz genau, welche Körperstellen dabei in Frage kommen, d. h. an welchen Stellen das Tier überhaupt ctwas fühlt und ihm Schmerzen zugefügt werden können. Das sind natürlich vor allem die mit Schleimhaut bedeckten Körperstellen, also die Lippen und das als Tast- und Greiforgan dienende äußerste Ende des Rüssels und weiterhin der After und die Geschlechtsöffnungen. Aber gerade an diesen Stellen darf cin Elefant auf keinen Fall gercizt oder gar verwundet werden. Daneben gibt es aber zahlreiche andere Stellen, sogenannte nila ${ }^{1}$, an denen der hendua angesetzt werden kann. In alten Palmblatthandschriften wird eingehend an Hand von Skizzen über diese nila berichtet, mit welchem Studium sich vor allem der Elefantenheilkundige (aliya vedarala) zu befassen hat. Einer dieser Schriften ist auch dic obenstehende Skizze entnommen, in der dic verschiedenen nila durch Punkte angedeutet sind (Abb. 3).

\section{Fähigkeiten und Verwendung}

Nach Ansicht aller Eingeborenen Ceylons ist der Elefant unbedingt das intelligenteste und gelehrigste Tier, welche Ansicht manche europäische Gelehrte nicht teilen. Wer jedoch jemals genau zugeschaut hat, wie ein abgerichteter Elefant seine Arbeit verrichtet, und wer vor allem jemals zusah, wie das Abrichten erfolgt, d. h. welche fabelhaft kurze Zeit dazu erforderlich ist, wird bestimmt positiv urteilen.

${ }^{1}$ Man versteht unter nila ganz allgemein die empfindlichen Stellen des Körpers, aber auch solche. an denen man den Puls fühlen kann. 
$\mathrm{Da}$ der Elefant auch ein außerordentlich gutes Gedächtnis besitzt, dürfie ebenfalls zur Genüge bekannt sein. Nicht aber trifft zu, daß ein gezähmter Elefant, dem es gelingt, die Freiheit zu erlangen, sich nie wieder fangen lassen wird. CORSE berichter von einem solchen Fall, wo ein gezähmter Elefant, anläßlich eines Elefantenfanges nach der Methode des Kraaling, mit andern wilden Elefanten zusammen in den Busch lief, aber einige Jahre später; abermals anläßlich eines Kraaling, eingefangen wurde. Ohnc Umstände ließ er zu, daß man ihn berührte und sich einer der Elefantenfänger auf seinen Rücken setzte. In kurzer Zeit war er, obschon er etliche Jahre völlig frei im Busch gelebt hatte, wieder vollkommen zahm und erfülle die Befehle des mahut wie zuvor.

Nach Ansicht der Eingeborenen soll dem Elefanten bloß das Sprachvermögen fehlen, im übrigen aber seine Intelligenz an dicjenige des Menschen heranreichen, bei welchem Urteil natürlich auch religiöse Ansichten mitspielen. Daß man mit ihm sprechen kann wie mit einem Menschen, das heißt, $\mathrm{da} \beta$ er alles, was man ihm sagt, auch wirklich verstcht, und daß man einen wilden Elefanten in sehr kurzer Zeit so weit bringen kann, daß er alles, was man von ihm verlangt, zu tun gewillt ist und auch tut, spricht zur Genüge dafür, daß man es bei ihm mit einem äußerst intelligenten Wesen zu tun hat. Besonders intelligente Elefanten, so behauptet der Eingeborene weiter, vermögen sogar mit ihrem Rüssel sinnvolle Figuren, Zeichen, ja selbst singhalesische Buchstaben in den Sand zu zeichnen, aus welcher Fähigkeit jeweils großes Aufsehen gemacht wird. Solche Tiere sind mehr als die andern geschätzt und werden sehr hoch bewertet.

Gelegentlich werden männliche Elefanten störrisch oder gar bösartig, welchen Zustand man must nennt. Dies hängt mit dem $\mathrm{Ge}$ schlechtsleben zusammen. Gewisse Drüsen des Kopfes sondern dann, wie schon R. KNox bemerkt, ein öliges Sekret ab, das zum Munde herausfließt. Wenn ein Tier in diesen Zustand gerät, muß es natürlich mehr als sonst überwacht werden, und man darf nicht zu viel Arbeit von ihm verlangen. Doch ist dieser Zustand immer vorübergehender Natur und betrifft nur männliche Tiere.

Oftmals versucht man, diesem Zustand auch mit cinem geeigneten yantra und mantra beizukommen. Erstgenanntes zeichnet man auf ein Stück Papier oder auf einen Palmblattstreifen, den man dem betreffenden Elefanten mit einem Leckerbissen, zum Beispiel einem Stück Palmzucker, zu fressen gibt; oder aber man macht mit einem scharfen Messer an einer wenig empfindlichen Stelle einen Schlitz in die Haut, steckt das yantra hinein und näht die aufgeschnittene Hautpartie wieder $\mathrm{zu}$, wobei man gleichzeitig ein geeignetes mantra spricht. Ein solches lautet zum
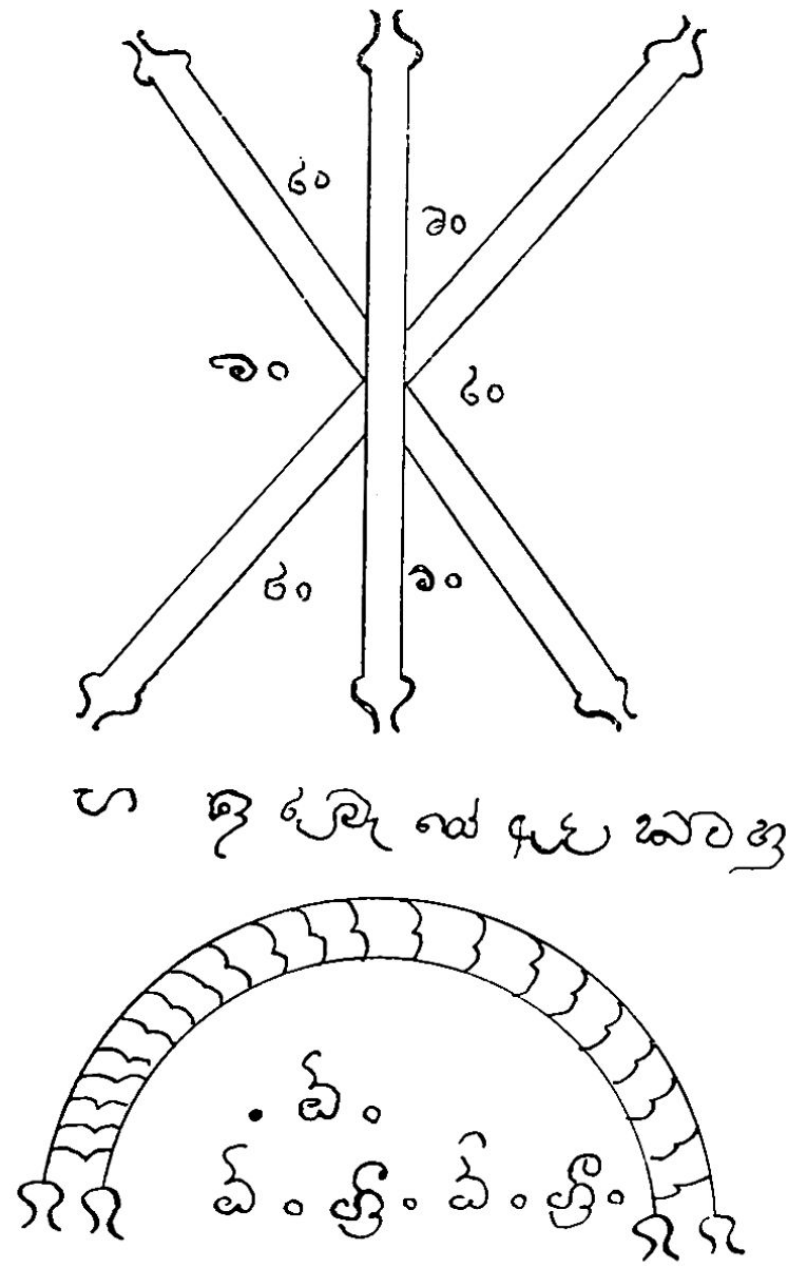

Ab̉b. 4. yantra zur Besänftigung störrischer Elefanten Beispiel: "Om srin ran ran ran Rama-rana nai-nai Kasita -sitita, sitita mutra-mutra; esvaha.» (Abb. 4.)

Zahlreich sind auch die Medizinen, die störrisch gewordenen Elefanten verabreicht werden; die Zusammensetzung wird jedoch meistens geheimgehalten. Jeder mahut besitzt seine Mittel und ist von deren Wirksamkeir unbedingt überzeugt. Aber auch cler Elefantenheilkundige weiß in diesen Dingen Bescheid, obschon es auch solche gibt, die mit dergleichen Praktiken nichts zu tun haben wollen. Eine solche Medizin besteht zum Beispiel aus den folgenden Bcstandteilen: Hinäti-hal, eine Art ungekochter Reis, den man zu Mehl stampft und dem man den Saft der attan-Wurzcl (Datura fastuosa L) und des Vishnu-kranta-Krautes (Evolvulus pusilius Choisy) sowie etwas Borax (pus kara) hinzufügt, so daß ein dicker Brei entsteht, den man an einem bestimmten Tage, bei zunehmendem Mond, dem Elefanten mit Palmzucker zusammen verabreicht.

Ein anderes Mittel ist in Wasser gelöster Borax, dem man die verbrannten Schopffedern cines Pfauen (monara kudimbiya) hinzufügt, welche Flüssigkeit man dem Tier zu trinken gibt. Oder aber man nimnt eine völlig ausgereiftc Koliosnuß, schlägt sie entzwei und spricht in die beiden Schalhälften das 
folgende mantra: "Om Namo, Sri Buddha Dyaya - dyaya; esvaha.» "Om Namo, nara yana namang namo anguli mala kive; svahang", und zwar $2848 \mathrm{mal}$. Hierauf wird der Kern der Kokosnuß mit einem Messer herausgeholt, zerkleinert, mit Palmzucker vermengt und dem Elefanten zu fressen gegeben.

Wirksamer wird diese Methode, wenn man gleichzeitig ein geeignetes yantra in Anwendung bringt, indem man dieses in einen Palmzuckerkuchen oder in die Schale ciner Frucht, zum Beispiel einer Bananc, einritzt, mit einem mantra bespricht und dem Elefanten hinreicht. Der Elefant wird sich dann sehr bald beruhigen und wieder völlig zahm und willig werden.

Die in einem Kral oder einzeln eingefangenen Elefanten werden in der Regel, bevor sie völlig gezähmt worden sind, an Privatpersonen oder Unternehmungen verkauft, die dann alles weitere selbst zu besorgen haben. Der Meistbietende setzt sich in den Besitz des oder der gewünschten Tiere, nicht ohne sich die Kaufobjekte genauestens anzusehen und sich über deren Alter, körperlichen Zustand und deren Leistungsfähigkeit zu orientieren, wobei er nötigenfalls eine Vertrauensperson zu Rate zieht. Eine solche ist in allen Fällen der Elefantenheilkundige.

Die meisten gezähmten Elefanten befinden sich heute im Besitz von Plantagenbesitzern oder Unternehmungen und von Privatpersonen, die solche Tiere halten, damit sie ihnen direkt oder mittelbar im Ausleihesystem eine ständige Einnahmequelle verschaffen. Zwvanzig Rupien ist die heute übliche Leihgebühr für einen Arbeitselefanten pro Arbeitstag, der allerdings nur aus fünf oder sechs Vormittagsstunden besteht. Nachmittags pflegt kein Elefant Arbeit zu verrichten. Die Elefanten bilden so eine Art Kapitalanlage, die sich unter Umständen gut vcrzinst. Auch die größeren Tempel Ceylons besitzen vielfach einen oder zwei Elefanten, die ein- oder zweimal im Jahre zu den festlichen Umzügen verwendet werden. Im übrigen sind diese Elefanten wie die andern zur Arbeit abgerichtet und werden auch vermietet.

Auch der ehemalige singhalesische König hielt an seinem Hof zahlreiche gezähmte Elefanten, teils aus Prestigegründen, teils zur Arbeit in seinen Pflanzungen und zum Zeitvertreib. Man ließ gecignete Tiere innerhalb eines umzäunten Platzes paarweise miteinander kämpfen, wie heute noch an vielen indischen Fürstenhöfen. Dies Schauspiel wurde regelmäßig zur Belustigung des Königs, seiner Angehörigen und des ganzen Volkes veranstaltet. Einer der königlichen Elefanten war noch zur Zeit des letzten Singhalesenkönigs speziell zur Hinrichtung der Verbrecher trainiert, wobei die zum Tode Verurteilten von diesem Tier zertrampelt und in Stücke gerissen wurden, und zwar alljährlich auf einem bestimmten Platz und an cinem zum voraus bekanntgegebenen Tage, so daß jedermann dem Schauspiel beiwohnen konnte.

Schon damals bildeten die auf Ceylon eingefangenen und gezähmten Elefanten einen wertvollen Handelsartikel, nicht bloß im Inland, sondern auch mit dem Ausland, und vor allem pfiegten sich die indischen Fürsten mit Vorliebe ceylonesische Elefanten für ihren Privatgebrauch zuzulegen, da diese Tiere, wie es hieß, am besten abgerichtet waren.

Späterhin wurden zoologische Gärten und Menagerien Europas und Amerikas gute Abnehmer.

\section{Behandlung der Elefanten}

Jeder gezähmte jungè oder alte Elefant besitzt seinen mahut, einen Mann oder Burschen, der über das Tier die Aufsicht auszuüben, es bei seiner Arbeit zu überwachen, für seine tägliche Reinigung und Nahrung zu sorgen hat. Auf ihm ruht somit große Verantwortung, die nur Leute einer bestimmten Kaste zu erfüllen vermögen und dazu berechtigt sind. Seit vielen Jahrhunderten üben sie diesen Beruf aus und haben Kenntnisse und Fähigkeiten erworben, die andere Leute nicht besitzen. Der Sohn lernt vom Vater und gibt seine Kenntnisse an seinen Sohn weiter; und so sind sie mit den Elefanten viel mehr vertraut als Leute irgendwelcher anderer Kasten, was offenbar auch die Elefanten merken. Schon die Art und Weise, wie die mahut mit ihren Tieren sprechen, ist apart. Sie bedienen sich dabei gewisser Redewendungen, die im gewöhnlichen Sprachgebrauch nicht vorkommen, möglicherweise einer andern Sprache entlehnt sind, jedoch von den gezähmten Elefanten verstanden werden.

Manchmal hat ein mahut den Elefanten, der ihm in Pflege gegeben worden ist, bereits als wildes, im Kral oder beim Einzelfang erbeutetes Tier kennengelernt und auch mit dessen Zähmung und Abrichtung zu tun gehabt, in welchem Fall er mit seinem Schützling von vorneherein vollkommen vertraut ist. Mit dem ihm anvertrauten Tier bleibt ein mahut sozusagen für sein Leben verbunden. Von früh bis spät gibt er sich mit ihm ab, sitzt auf seinem Rücken, wenn es seine Arbeit zu verrichten hat, schrubbt es, wenn es beim Baden ist, sieht zu, daß es täglich seine Nahrung erhält, und verbringt die Nacht in seiner Nähe. 
Als Entlöhnung erhält in der Regel der mahut, so er einen auszuleihenden Elefanten betreut, 25\% der Einnahmen, also etwa fünf Rupien pro Arbeitstag; dafür aber muß er für alle Auslagen selbst aufkommen. Für die Nahrung des Elefanten sorgt in den meisten Fällen derjenige, der das Tier gemietet hat.

Normalerweise ist der gezähmte Elefant das gutmütigste Tier der Welt. Niemals wird er seinem mahut etwas zuleide tun, und bereitwilligst erfüllt er jeden Befehl und jede Aufforderung seines Gebieters. Dieses Gehorchen hat aber, vor allem, wo es

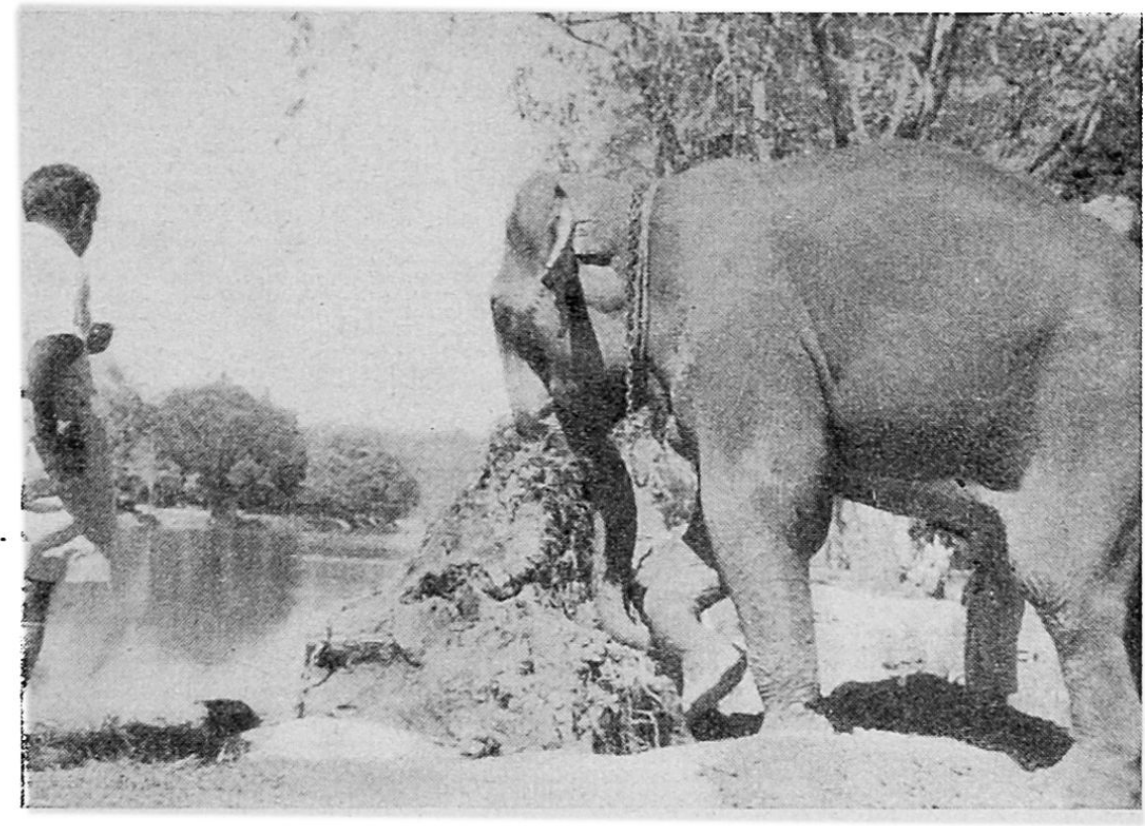

Abb. 5. Entfernung einer großen Baumwurzel durch Stoßen mit dem oberen Teil des Rüssels. Aufnahme P. WIRz sich um Arbeit handelt, eine bestimmte Grenze.

Jeder Elefant weiß genau, daß die Arbeitszeit für ihn, so wie die Sonne in den Zenit rückt, beendet ist. Darnach nützt alles Aufihneinreden nichts mehr. Jeder mahut würde daher auf unüberwindlichen Widerstand stoßen und unter Umständen gar Gefahr laufen, daß das Tier bockbeinig wird und auf alle Fälle zu seinem Recht zu kommen sucht. Kein Elefant wird also während der Nachmittagsstunden auch nur die leichteste Arbeit verrichten. Dies ist für ihn die Zeit der Erholung. Sowie die Mittagsstunde vorüber ist, werden die Arbeitselefanten von ihrem mahut an einen Fluß geführt, wo sich die Tiere sogleich ins Wasser begeben und niederlegen, während die mahut sich anschicken, deren Körper mit Kokosschalen abzuschrubben. Erst bei Sonnenuntergang treten sie den Rückweg an, wobei ein jedes Tier ein großes Bündel Grünzeug oder den Stamm einer kitul-Palme (Caryota urens L) mit sich trägt, mit dessen Vertilgung es den Abend und die erste Hälfte der Nacht verbringt. Das Mark der genannten Palme bildet einen Leckerbissen für den Elefanten, der es geschickt mit seinem Greiforgan herausholt, nachdem er mit den Füßen den Stamm aufgebrochen hat. Der größte Leckerbissen aber ist für ihn der aus dieser Palme gewonnene Zucker wie auch der frische oder gegorene Palmwein, den man einem Arbeitselefanten hin und wieder verabreicht, um ihn bei guter Laune zu halten oder wenn man eine besonders schwierige oder heikle Arbeit von ihm verlangt.

Der Elefant pflegt nur wenige Stunden ohne Unterbrechung zu schlafen, und zwar vornehmlich während der zweiten Hälfte der Nacht. In der Wildnis geschieht dies meistens stehend, gegen einen Baum gelehnt, seltener liegend, und nicht anders verhält es sich auch bei den gezähmten Tieren. Auch während des Badens im Fluß schläft der Elefant manchmal für kurze Zeit, wobei er, wie fast alle Säugetiere, die Augen schließt.

Der Lebenszyklus des Elefanten stimmt nach den Singhalesen und Tamilen mit demjenigen des Menschen überein, und so ziehen sie zwischen den beiden die weitgehendsten Parallelen.

Hinsichtlich des Sterbens der Elefanten wird manches behauptet, was jeder Grundlage entbehrt. Dahin gehört zum Beispiel die Geschichte von den Elefantenfriedhöfen. Ein Singhalese oder Tamile wird lachen, so man ihn danach befragt. Es heißt jedoch, daß der Elefant, wenn er alt geworden ist, genau weiß, daß es in absehbarer Zeit mit ihm zu Ende gehen wird. Fühlt er sein Ende herannahen, so sondert er sich von der Herde ab und begibt sich an einen einsamen Ort, wo er verendet. Wenn irgendwie möglich, sucht er zum Sterben einen Ort auf, wo Wasser vorhanden ist; denn wenn er auch, dem Tode nahe, keine Nahrung mehr zu sich nimmt, so kann er doch ohne Wasser nicht einen Tag leben. 


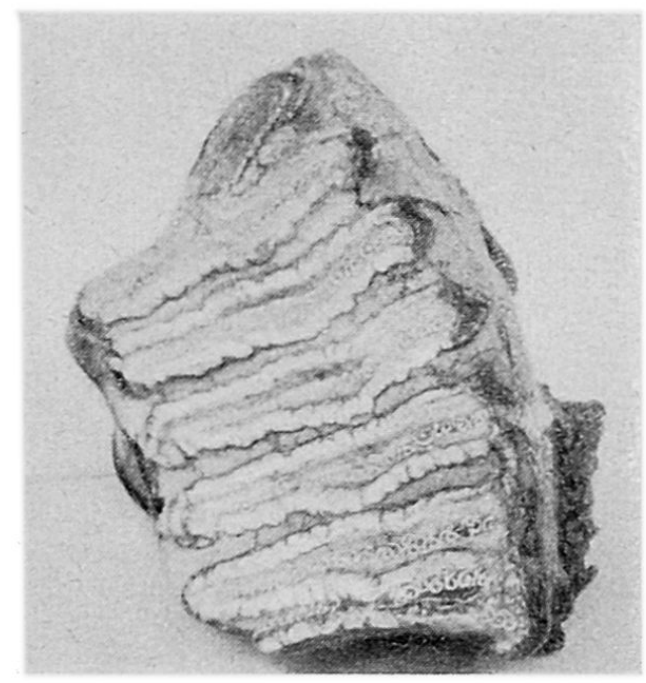

Abb. 6. Mit singhalesischen Schriftzeichen beschriebener Backenzahn eines Staatselefanten aus dem Kandy-Museum. Die Inschrift lautet in der Übersetzung folgenderweise: "Am Montag, am siebenten Tage, in der dunklen Hälfte des Monats Poson, im Jahre 1623 (1701 nach unserer Zeitrechnung) der Saka-Aera, genannt Vrasa; dies ist das Stück cines Zahnes, welches aus der rechten Kieferseite aus dem Munde cines weiblichen Elefanten herausfiel, welcher teilzunehmen pflegte an den staatlichen Reisen.»

Weshalb aber, so wird man fragen, findet man so selten Knochen von verendeten Elefanten und niemals auch nur ein Stückchen Elfenbein? Auch diese Frage ist leichter zu beantworten, als man glaubt. Erstens verkriecht sich der Elefant, wenn er stirbt, in den dichten Dschungel; zweitens sorgen die andern Tiere der Wildnis, vor allem das Stachelschwein und andere Nager, und weiterhin die Feuchtigkeit und die niederen Pflanzen dafür, daß selbst in kurzer Zeit von den Knochen eines veiendeten Elefanten nichts mehr übrigbleibt. Ein an der Oberfläche liegender Kadaver verschwindet bekanntlich in den Tropen in kurzer Zeit restlos.

Der verendete, gezähmt gewesene Elefant wird sogleich an Ort und Stelle, und zwar so tief wie möglich, begraben, nachdem man erst den Schwanz (ali valga), oder doch wenigstens die Schwanzhaare, abgeschnitten hat, aus welchen man Armringe als Amulette verfertigt. Auch ein Stück der Haut (ali hang) wird häufig aus dem Kadaver herausgeschnitten, da diese ein wertvolles Medikament abgibt. Dem Rauch verbrannter Elefantenhaut schreibt man antiseptische Wirkung zu, weshalb man schwe:heilende, eiternde Wunden damit zu behandeln pflegt. Manchmal wird auch ein Backenzahn (ali dat) aus dem Ober- oder Unterkiefer herausgeschlagen; denn auch dieser gibt ein Medikament ab. In einem Mörser zu Pulver zerrieben und mit dem Saft einer kleinen Zitrone (dehi) vermengt, ist er ein Mittcl gegen Parotitis. Und schließlich findet - doch betrifft dies das lebende Tier - der Urin (ali mutra), mit Kräutern vermengt, in der singhalesischen Heilkunde Verwendung. Daß auch die Stoßzähne aus dem Oberkiefer herausgeholt werden, versteht sich wohl von selbst. Den übrigen Kadaver läßt man hingegen intakt, begräbt ihn also unzerteilt ${ }^{2}$.

\section{Elefantenheilkunde}

Obwohl der gezähmte ceylonesische Elefant sich nicht vermehrt und somit nicht gezüchtet werden kann, also auch nicht zu den eigentlichen Haustieren zu rechnen ist, bedarf er doch wie diese der Pflege. Man verlangt von ihm Arbeit, verabreicht ihm Nahrung, die wohl nicht immer der Dschungelnahrung entspricht, und gewöhnt ihn an einen gewissen Lebensrhythmus, den er sich zu eigen macht, ohne zu revoltieren, der aber von demjenigen der Dschungelelefanten sehr verschieden ist. Das alles birgt gewisse Gefahren, vor allem die Gefahr, gewissen Krankheiten zum Opfer zu fallen, wie sie beim Dschungelelefanten nicht oder doch viel weniger häufig auftreten. Dieser letztgenannte kennt so gut wie keine Feinde. Außer dem Tiger, der jedoch auf Ceylon nicht vorkommt, vermag ihm kein Tier etwas anzutun. Schlangen, selbst die so gefährliche Polonga (Russel-Viper), vermögen die dicke Haut seines Körpers nicht zu durchbeißen. Ebensowenig vermögen ihm stechende Insekten etwas anzutun. Es bleiben höchstens Parasiten, die das Gewebe der Eingeweide zerstören. Sein wirksamster Feind ist der Mensch, der von jeher auch diesem großen Tier der Wildnis nachstellt. Die

2 Dem Blut des Elefanten wird jedoch weder auf Ceylon noch in Indien und Hinterindien eine besondere Eigenschaft, weder in therapeutischem noch in irgendeinem andern Sinne zugeschrieben. Das Entnehmen von Blut aus dem lebenden Tierköriper würde auch die buddhistische Religion nicht zulassen. 
Gefahren, denen der gezähmte Elefant unterworfen ist, sind teils durch die veränderte Lebensweise, teils durch die von ihm geforderte Arbeitsleistung bedingt. Schlimmere Gefahren bedingen veränderte Lebensweise und Ernährung. Trotz seiner Größe und seiner allen äußeren Einflüssen widerstehenden dicken Haut muß der gezähmte Elefant mit Sorgfalt behandelt werden. Kleine Fütterungs- und Behandlungsfehler können sich bitter rächen.

Von alters her kennt man sowohl in Indien wie auch auf Ceylon Elefantenärzte, die genau Bescheid wissen, wie mit einem gezähmten Elefanten umgegangen werden muß und welchen Krankheiten er unterworfen ist. Entsprechend dem Heilkundigen, der sich mit der Behandlung erkrankter Menschen befaßt, wird der Elefantendoktor ebenfalls vedarala oder vielmehr aliya-vedarala (aliya = Elefant; vedarala von veda, das Wissen) genannt. Die vedarala bilden keine besondere Kaste. Sie schöpfen ihr Wissen teils aus Büchern (Palmblatthandschriften und gedruckten Büchern), teils von Lehrmeistern und lernen, genau wie unsere $\ddot{A}$ rzte, während ihrer Praxis unablässig hinzu. Sehr oft wird der Sohn eines vedarala wiederum ve. darala und übernimmt somit das Wissen von seinem Vater, der ihm alle Geheimnisse der Heilkunst schon von klein auf beibringt. So angesehen der Beruf eines aliya-vedarala ist, so gibt es heute doch nur noch sehr wenige Elefantenheilkundige.

Ein solcher weit bekannter Mann lebt in der Nähe von Katugastota, vier Meilen nördlich von Kandy. Manickrala ist sein Name. Sein Vater übte ebenfalls diesen Beruf aus und wurde 102 Jahre alt. Er war äußerst angesehen und hatte in seinen Jugendjahren am Hofe des letzten Singhalesenkönigs mit dessen Elefanten zu tun gehabt. Auch Manickrala ist sehr angesehen. Er hat in seinem Beruf sehr vicl zu tun; denn unter den vielen Arbeitselefanten, die in seiner Gegend gehalten wcrden, ist häufig ctwas nicht in Ordnung, und wenn jemand einen Elefanten kauft, so wendet er sich zunächst an Manickrala, damit er

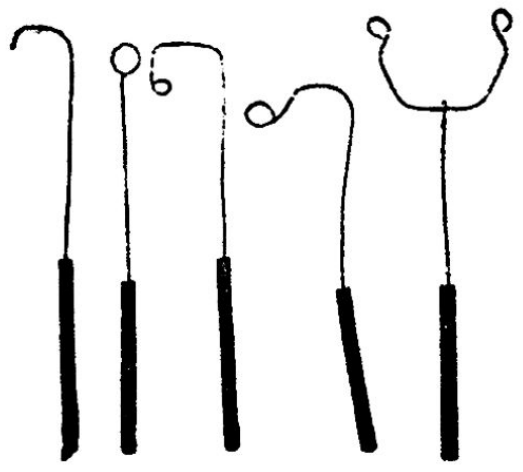

Abb. 7. "Brenneisen" zur Behandlung eines an Rheuma leidenden Elefanten. Zirka $1 / 4$ der natürlichen Größe. Aufnahme P. WIRZ den in Frage kommenden Elefanten in Augenschein nehme und sein Gutachten abgebe. Natürlich verdient er gut.

Wie der Schmied, der dic Elefantenstachel anfertigt, so pflegte, wie cs scheint, auch der aliyavedarala in früherer Zeit die Elefantengottheit Gancsha als scinen Schutzpatron zu betrachten. Der Beruf des aliya-vedarala hatte also früher mehr denn heutc eine stark betonte kultische Scite. Wie der Hersteller des Elefantenstachels, so hatte jedenfalls auch der aliya-vedarala bei bestimmten Anlässen der Elefantengottheit ein Opfer anzubieten, damit seine Heilmittel und Heilmethoden Erfolg hatten.

In der Nähe der Behausung des genannten aliya-veda rala steht heute noch ein sehr altes devalaya (Tempelchen). Unter den zehn Gottheiten, die daselbst verehrt werden und auf bemalten Holzschilden, sogenannten petikandà, dargestellt sind und deren Attribute oder Waffen daneben ihren Platz haben, nimmt die Elefantengottheit Ganesha cine bevorzugte Stellung ein. Diesen Gottheiten werden wöchentlich, anläßlich einer kleinen halbnächtlichen Zeremonie, an der auch der aliya-vedarala teilnimmt, durch den Priester (kapua), dem das devalaya unterstellt ist, Opfer angeboten. Zwischen dem aliya-vedarala und der Elefantengottheit besteht somit von alters her ein enger Kontakt, und ersterer vcrmeinte, daß cr sein Wissen und Können aus göttlicher Quelle empfangen habe.

Der Elefantenheilkundige kennt verschiedene Methoden der Behandlung, die jeder, der diesen Beruf ausübt, unbedingt beherrschen muß. Die eine besteht in der Anwendung rein äußerer Hilfsmittel, wobei u. a. gewisse Stellen des Körpers mit glühendgemachten Eisendrähten betupft werden. In Abb. 7 ist eine Anzahl solcher «Brenneisen» (nila pussana katu) wiedergegeben. Jedes Instrument ist mit einem hölzernen Griff versehen, und das andere Ende des Drahtes ist zu einem Ring oder Haken geformt. Auch Augenleiden, wie Trübungen der Hornhaut, werden auf diese Weise 
behandelt, indem ein heißgemachter Ring, größer als das Auge, aufgelegt wird. Dieser besteht jedoch nicht aus Eisen, sondern aus einer Legierung verschiedener Metalle, deren Zusammensetzung geheimgehalten wird.

Eine andere Methode arbeitet mit Injektionen, wobei mit Hilfe einer gewöhnlichen Injektionsspritze (vastiya) gewisse Ingredienzien in das Muskelgewebe eingespritzt werden. Ein aliya-vedarala versteht sich auch auf operative Eingriffe, wobei er sich eines scharfen Messers (pihiya) sowie einer Schere (katura), in gewissen Fällen auch einer Zange (anduva) bedient. Solche Eingriffe sind naturgemäß mit größter Vorsicht auszuführen; doch haben sie meist keinen allzugroßen Erfolg. Sie werden daher nur im Notfall ausgeführt. Demgegenüber ist die äußere und innere Anwendung von Heilmitteln von allergrößter Bedeutung. Läßt der Gesundheitszustand eines Elefanten zu wünschen übrig, so äußert sich dies vor allem darin, daß das Tier keine Nahrung mehr zu sich nimmt, sein Stuhlgang nicht normal ist oder Würmer aufweist und das Tier vorwiegend auf der Erde liegt, sich nur ungern erhebt und nicht gewillt ist, Arbeit zu verrichten. Man unterläßt es natürlich, einen erkrankten Elefanten zur Arbeit anzuhalten oder frei herumlaufen und ihn sein tägliches Bad nehmen zu lassen. Man hält ihn, wenn es irgendwie angeht, in der Nähe des Hauses, wo man ihn ständig beobachten und beaufsichtigen kann.

Ein ernsthafter vedarala beobachtet den Puls des erkrankten Tieres, betrachtet die Beschaffenheit der Fäkalien und des Urins, die Schleimhaut von Mundhöhle und Rachen, die Augen und anderes mehr. Weisen die Schleimhaut des Mundes und die Zunge einen Belag auf, ist der Atem übelriechend, der Kot und Urin anormal, das Auge trüb oder gerötet, so sind di=s unfehlbare Anzeichen, daß eine Behandlung notwendig ist.

Zur Behandlung äußerer Wunden und Geschwüre wird folgendes Mittel empfohlen: Man nehme gleiche Mengen von Gelbwurz (kaha), Blätter und Rinde des kohomba-Baumes (Swertia Chirata Buch) und Weihrauch (kattakumanchal), zerkleincre alles in einem Mörser, füge etwas Honig hinzu und verbrenne die Mischung in einem Kohlenbecken, worauf man den Rauch über dic Wunden streichen lasse. - Hicrauf behandle man dic W'unden mit dem Brei, der durch Zerstampfen folgender Kräuter gewonnen wird: Gelbwurz (kaha), Blätter und Rinde des gu rulla-Baumes (Leea sambucina Blanco), Blumen des batu- und totilla-Baumes (Solanum indicum L, Spathodea indica Pers.) sowie die Stengel und Blüten des Schlinggewächses ela-katayrolu (Clitoria Ternatea L).

Oder aber man nehme ungefähr eine Milchbüchse voll Honig und Frauenmilch, füge eine kleine Menge zu Pulver zerriebener tipili-Samen (Piper longum L) und valanga-sal-Samen (Embelia spec.) sowie einc Milchbüchse voll Rahm von gekochter Milch (ghi) und ctwas Stcinsalz (sahinda lunu) hinzu. Alles ist zusammen in einen Topf zu tun und längere Zeit zu erhitzen, bis ein mäßig dicker Brei entsteht, mit dem, nachdem er kalt geworden ist, die wunden Stellen zu bestreichen sind.

Bei eiternden Wunden und Geschwüren wendet man einen Brei aus zerstampften tala-Samen (Sesamum spec.), ulu-hal (Trigonclla foenum graecum L), Samen von enderu (Ricinus communis L) und solche von kaluduru (Nigella indica Roxb.) an. Oder man zerstampft gleiche Mengen von Mung-Samen (Phascolus Mungo Wall.), ulu-hal (Trigonella focnum graecum L), komarika (Aloc indica Royle) und geadopala (Portulaca oleracea L), kocht das Gemisch längere Zcit unter Zusatz von W'asser, fügt den Saft einer Citrusart (ambul dodang) hinzu, bis ein mäßig dicker Brei entsteht, den man erkalten läßt.

Häufig leiden junge Tiere an mehr oder weniger bösartigen Geschwüren am Hals, wogegen ebenfalls eines der beiden letztgenannten Mittel angewendet wird.

Bei alten Tieren werden oft dic Fußsohlen rissig. Sie werden mit Teer oder mit kohomba-tel, dem Öl, das man aus den Samen von Swertia Chirata Buch bereitet, bestrichen.

Ältere gezähmte Elefanten leiden nicht selten an Rheuma. Ob dies auch für den Dschungelelefanten gilt, weiß man nicht; doch ist anzunehmen, daß die veränderte Lebensweise und dic geringere Bewcgungsmöglichkeit beim gczähmten Elefanten etwas ausmachen. Ein Elefant, der an Rheuma leidet, ist schlecht gelaunt, weigert sich zu arbeiten und oftmals auch Nahrung zu sich zu nehmen. Man sucht diesem Leiden entgegenzutreten, indem man die empfindlichen Stellen, besonders die nila, mit glühendgemachten "Brenneisen» berührt. Daneben verabreicht man Medikamente. Ein Rezept lautet: "Man löst etwas Kochsalz in Wasser auf, fügt feingeschnittenen Ingwer, junge, ebenfalls zerkleinerte Arekanüsse und Samen der kalu -a ralu - Pflanze (Terminalia Chebula Retz) hinzu, läßt das W'asser längere Zeir kochen, filtricrt durch ein Tuch, fügt weiteren Ingwersaft hinzu und gibt dem Elefanten die Flüssigkeit zu trinken. Oder man nimmt a ralu-Samen (Terminalia Chebula Retz), Früchte des bulu- und nelli- 
Baumes (Terminalia Belerica Roxb. und Emblica offic. Gaertn.), Rinde des kohomba-Baumes (Swcrtia Chirata Buch), Gelbwurz, katu-korasana (Picrorrhiza Kurroa Royle), rasa-kinda (Myristica offic. L), väl-madata (Rubia Munjista Roxb.), venivälla (Allmania nodiflora R. Br.) — von allem gleiche Gewichtsmengen, tut alles in einen irdenen Topf mit Wasser und bereitet eine Abkochung, die man dem Elefanten während dreier Tage zu trinken gibt. Oder aber man nimmt gleiche Mengen von rasakinda (Myristica offic. L), Früchte des nelli-Baumes (Emblica offic. Gaertn.) und der Tamarinde (Tamarindus indica L), tut alles in einen Topf mit Wasser und bereitet eine Abkochung, fügt Zucker hinzu und läßt abkühlen. Gleichzeitig bestreicht man den Kopf des Tieres mit einem wie folgt bereiteten Brei: Man nimmt die Milch und das Fruchtfleisch einer jungen Kokosnuß, fügt koma rika (Aloe indica Royle) sowie feingeriebenes Sandelholz hinzu, bringt alles in einen Topf mit etwas Wasser und läßt längere Zeit kochen; hierauf fügt man Kuhurin, Kuhmist und Rahm von gekochter Milch (ghi) in gleichen Mengen hinzu und läßt das Gemisch nochmals kochen. Während dreier Tage wird der Elefant auf diese Weise behandelt. Hierauf läßt man ihn ein Bad nehmen.»

Ein Elefant bekommt häufig Fieber. Auch in diesem Falle bestreicht man den Kopf und häufig auch den Rüssel des Tieres mit einem aus verschiedenen Kräutern bereiteten Brei, wie bei fiebernden Menschen. Ein weiteres Breirezept ist das folgende: Ingwer, Knoblauch, Stengel der velmi-Pflanze (Berberis aristata D. C.), Senfsamen, Pfeffersamen, Samen von karalsebo (Achyranthes aspera L), Knollen von vada-kaha (Acorus calamus L), Rinde des murunga-Baumes (Moringa pterygosperma Gaertn.), von allem gleichviel, wcrden in eincm Mörser zerrieben und mit Sesamöl erhitzt.

Störungen in den Verdauungsorganen sind nicht minder häufig. Sie äußern sich darin, daß das Tier kcine Nahrung zu sich nehmen will, sich gereizt benimmt, auf dem Boden liegt, Sand oder Erde frißt.

Der aliya-vedarala bereitet dagegen aus folgenden, in einem Mörser oder auf einem flachen Stein zerriebenen und miteinander vermengten Bestandteilen einen Brei, der dem Elefanten verabreicht wird: Von Blättern des kohomba-Baumes (Swertia Chirata Buch), des povatta-Baumes (Adhatoda vasica Nees), Pfeffersamen (Piper nigrum L), Knoblauch (sudulunu), Salpeter (sahinda-lunu) werden gleiche Gewichtsmengen genommen; doch dürfen die Blätter weder zu jung und zart noch zu alt oder gar dürr sein. Hierauf wird der Brei zu kleinen Kugeln geformt und jede cinzelne in eine Apfelsine oder ein Stück Palmzucker gesteckt, dem Elefanten in den Mund geschoben. Dies wiederholt man regelmäßig während etlicher Tage.

Zahlreich sind die Mittel, die bei Augenleiden angewendet werden. Solche sind sehr häufig. Am schlimmsten dürften Trübungen der Cornea sein, die man, wic erwähnt, durch Auflegen heißgemachter Metallringe zu bescitigen sucht. Eines der am häufigsten, auch beim Menschen angewendeten Mittel ist Frauenmilch, der man den Saft der polpala-Pflanze (Aerva lanata Juss) beimischt. Man zerreibt auch die Früchte des aralu-Baumes (Terminalia Chebula Retz) und die Stengel der velmi-Schlingpflanze (Berberis aristata D.C.), bringt beides in ein Tuch, erhitzt es im W'asscrdampf und preßt den Saft aus, den man in die Augen träufelt.

In schlimmeren Fällen nimmt man zerkleinerte a ralu-Früchte, einige feingeschnittene Knoblauchknollen, fügt etwas Alaun (sinakkarang) und etwas Saft einer kleinen Zitronc (dehi) hinzu, erhitzt alles in einem Topf, bis ein mäßig dicker Brei entsteht, den man nach dem Erkalten rings um die Augen streicht.

Man bereitet auch einen Brei aus verbranntem Reis (käkul bat), den Früchten des a ralu-und bulu-Baumes und Lampenruß, wobei man von jeder Substanz ungefähr dic gleiche Gewichtsmenge nimmt, fügt etwas Honig hinzu und streicht das Gemisch rings um die Augen.

Ein Mittel gegen Augenleiden aller Art ist auch das folgende: Früchte des a ralu- und bulu -Baumes werden mitsamt den Wurzeln der nelli- und babila-Pflanze (Emblica offic. Gaertn. und Sida acuta Burm), der Rinde des beli-Baumes (Acgle marmelos Cerre) und des rasa-kinda-Baumes (Myristica offic. L) sowie des iri-veriya-Strauches (Plectranthus zeylanicus Benth.), nachdem alles zerkleinert worden ist, längere Zeit in Wasser gekocht, worauf man den erkalteten Abguß mit Honig, dem Rahm von gekochter Milch (ghi) oder cinem Öl vermengt und dem Tier zu trinken gibt.

In leichtern Fällen genügt ein aus zerriebenen Lotos- oder Seerosenblättern (tamburu) und dem Rahm von gekochter Milch (ghi) oder ein aus Alaun mit etwas Opium und der Asche von Haaren mit etwas Zitronensaft bereiteter Brei, der in gleicher Weise, doch auf cinem Eisenblech oder der Klinge ciner Hacke erhitzt, angewendet wird.

Zahndefekte sollen, wic mir von verschicdenen Seiten versichert wurde, bei Elefanten so gut wic nie vorkommen, und niemals hat der aliya-vedarala etwas damit zu tun. Es kommt auch bei einem alten Elefanten nicht zur vollständigen Abnützung der Kauflächen, wie gelcgentlich bei den in den zoologischen Gärten gehaltenen Elefanten, wo das Tier schließlich überhaupt nicht mehr zu kauen vermag und an Magen- oder Darmkrankheiten zugrunde geht. Daß diese mit der Verabreichung ungeeigneter Nahrung beziehungsweise dem Mangel an gewissen Aufbaustoffen oder Vitaminen zusammenhängt, ist mit Sicherheit anzunehmen. Auf Ceylon weiß man jedoch nichts darüber zu berichten.

Literatur. Aliya védita (Palmblatthandschrift), o. J. - BArrow, Sir G.: Ceylon Past and Present (London 1873). - Bennet, J. W.: Ceylon and its Capabilitics (London 1848). - Brooke, Ellior C.: The real Ccylon (Colombo), o. J. - CAve, H. W.: The Book of Ceylon (London 1908). - CoARSE, J.: 
Observations on Habits and Manners of Elephants (Philosoph. Transact. of the Royal Soc., I.ondon 1799). - Ewers, H. H.: Indien und ich (München 1917). - Forbes, H.: Eleven Years in Ceylon, 2 vol. (London 1840/41). - Fowkre, Ph.: The King of Beasts («Daily News», Colombo, 10. Febr. 1940). Geiger, W.: Ceylon. Tagebuchblätter und Reiseerinnerungen (Wiesbaden 1898). - GunUwARD.ANA, G.W.G.: Dravya Guna Nighantuwa and Auwsadha Nama Aracadia or Illustr. Encycl. of Tropical Plants and Drugs (Colombo 1912). - KNOx, R.: Ceylanische Reisebeschreibung oder historische Erzählung von der in Ostindien gelegenen Insel Ceylon (Leipzig 1689). -- Perer., M. D. E.: Gajavoga Sathakaya (Treatment of Diseases of Elephants; Kandy 1918). - Scridu, S. : Das Gebiß der Elephanten (Verhandlungen der Naturforschenden Gesellschaft, Basel, Bd. LIX, 1948). — Schмidr, E. : Ceylon (Berlin 1897). - Spitrel, R. L.: Wild Ceylon (Colombo 1924). - Ders.: Far off Things (Colombo 1933). Tennfent, J. E.: Ceylon, 2 vol. (London 1859/60). - Willis, J. C.: Handbook of Ceylon (Colombo 1907). - Ders.: A revised catalogue of the flowering plants and ferns of Ceylon (Colombo and London 1922).

\title{
LES ÉLÉPHANTS DE CEYLAN
}

Parmi tous les animaux domestiques des Cingalais et des Tamiles de Ceylan, l'éléphant domestiqué occupe une position particulière. Sa capture ainsi que le dressage de ce pachyderme fait sans doute partie de la culture et la tradition hindoue. Les veddas, les plus anciens habitants, ne connaissaient pas ces usages. La science des guérisseurs d'éléphants spécialement instruits se base sur de très vieilles traditions, connus de très peu d'indigènes. A ce sujet, bien des détails sont notées en vieilles écritures à main sur des feuilles de palmier.

\section{GLI ELEFANTI DI CEYLON}

Fra tutti gli animali domestici dei Cingalesi e dei Tamili di Ceylon, l'elefante domestico occupa una posizione speciale. La cattura e l'addomesticamento di questo pachiderma fa senza dubbio parte della tradizione e della cultura indiana. I Vedda, gli abitanti ceylonesi più antichi, non avevano affatto conoscenza di queste pratiche. Di speciale interesse c̀ la scienza della guarigione degli elefanti, conosciuta soltanto da gente appositamente istruita, la quale scienza risale a vecchie tradizioni. Ben pochi sanno cose precise in merito e le maggiori conoscenze ci vengono trasmesse da scritture a mano incise su foglie di palma.

\section{SUDMAROKKO}

\author{
Umrisse einer Landeskunde \\ Von Paul Köchli
}

Als Südmarokko werden auf Grund orographischer Überlegungen die Landschaften südlich des Hohen Atlas bezeichnet. Diese Gebirgsschranke brachte es mit sich, daß größere südmarokkanische Gebiete erst verhältnismäßig spät unter französische Herrschaft gerieten, so ganz besonders der Streifen zwischen dem Oued Draa und dem Oued Ziz, den Djebel Sarho einschließend, wo die berberischen Stämme der Yafelmane und der Atta bis 1933 Widerstand leisteten.

Eine deutliche Grenze gegen dic eigentliche Sahara kann im Westen durch das Tal des Oued Draa südlich des Anti-Atlas gezogen werden. Im mittleren und östlichen Teile verliert sie sich in den saharischen Kies- und Sandwüsten. Zwischen den einzelnen Landschaften erheben sich in der Höhenentwicklung stark unterschiedliche Gebirgszüge, die je nachdem eine mehr oder weniger ausgeprägtc Wasserscheide formen. So trennt der jungvulkanische Djebel Siroua $(3304 \mathrm{~m}$ ) das Sousgebiet vom Wadi Draa und seinem Einzugsgebiet. In seinem südlichen Teil umschließt er das klcine, selbständige Becken von Tazenakht $(1600 \mathrm{~m})$, bewohnt vom Stamme der Zenaga. Trotzdem darf die eigentliche Wasserscheide mit dem Tizi n'Taratine $(1950 \mathrm{~m})$ westlich von Tazenakht angenommen werden, wo sie durch ein kleines Hochplateau zwischen den Ortschaften Ighghi und Kourkouda gebildet wird. Aus dem Bekken von Tazenakht führt der $1700 \mathrm{~m}$ hohe $\mathrm{Paß}$ n'Bachkoun ins eigentliche Flußgebiet des Draa.

Bedeutend weniger auffallend ist die Wasserscheide zwischen dem Wadi Draa und dem Tafilalet. Sie verläuft zwischen dem Einzugsgebiet des Oued Dadès, des wichtigsten Zuflusses des Draa, und dem Oued Todra, dem bedeutendsten Nebenfluß des Oucd Ziz, in einer schwach gewellten Hochfläche in zirka $1600 \mathrm{~m}$ Höhe, östlich der Ortschaft Boumalne, das auf dem wichtigsten Verbindungswege zwischen den beiden Landesteilen liegt. Auch hier breitet sich an der Wasserscheide ein kleines, selbständiges Bccken aus, das von Foum el Kous in $1430 \mathrm{~m}$ Höhe, während das westlich davon gelegenc 